\title{
About the detectability of glycine in the interstellar medium
}

\author{
M. Lattelais ${ }^{1}$, F. Pauzat ${ }^{1}$, J. Pilmé ${ }^{1}$, Y. Ellinger ${ }^{1}$, and C. Ceccarelli ${ }^{2}$ \\ 1 UPMC Univ. Paris 06, UMR - CNRS 7616, Laboratoire de Chimie Théorique, 75005 Paris, France \\ e-mail: pauzat@lct.jussieu.fr \\ ${ }^{2}$ Laboratoire d'Astrophysique, Observatoire de Grenoble, 38041 Grenoble, France
}

Received 1 November 2010 / Accepted 12 May 2011

\begin{abstract}
Context. Glycine, the simplest of aminoacids, has been found in several carbonaceous meteorites. It remains unclear, however, wether glycine is formed in the interstellar medium (ISM) and therefore available everywhere in the Universe. For this reason, radioastronomers have searched for many years unsuccessfully to detect glycine in the ISM.

Aims. We provide possible guidelines to optimize the return of these searches. Since, for most of the species observed so far in the ISM, the most abundant isomer of a given generic chemical formula is the most stable one (minimum energy principle (MEP)), we assess whether neutral glycine is the best molecule to search for or whether one of its isomers/conformers or ionic, protonated, or zwitterionic derivatives would have a higher probability of being detected.

Methods. The question of the relative stability of these different species is addressed by means of quantum density functional theory (DFT) simulations within the hybrid B3LYP formalism. Each fully optimized structure is verified as a stationary point by means of a vibrational analysis. A comprehensive screening of 32 isomers/conformers of the $\mathrm{C}_{2} \mathrm{H}_{5} \mathrm{O}_{2} \mathrm{~N}$ chemical formula (neutral, negative, and positive ions together with the corresponding protonated species and the possible zwitterionic structures) is carried out. In the sensitive case of the neutral compounds, more accurate relative energies were obtained by means of high level post Hartree-Fock coupled cluster calculations with large basis sets (CCSD(T)/cc-pVQZ).

Results. We find that neutral glycine is not the most stable isomer and, therefore, probably not the most abundant one, which might explain why it has escaped detection so far. We find instead that $\mathrm{N}$-methyl carbamic acid and methyl carbamate are the two most stable isomers and, therefore, probably the two most abundant ones. Among the non-neutral forms, we found that glycine is the most stable isomer only if protonated or zwitterionic if present in interstellar ices.

Conclusions. Assuming that MEP can be applied to optimize our search for glycine, our conclusion is that this search will remain extremely difficult with the present instruments and we propose searching instead for other examples among the most stable isomers.
\end{abstract}

Key words. ISM: molecules

\section{Introduction}

Among all the molecules that could possibly be formed in the interstellar medium (ISM), $\alpha$-amino acids are arguably the most important pre-biotic ones, because they are critical to life as we know it on Earth.

Amino acids are molecules containing both amine and carboxyl functional groups. The family of the $\alpha$-amino acids have the amine $\left(-\mathrm{NH}_{2}\right)$ and the carboxyl $(-\mathrm{COOH})$ groups attached to the same carbon atom, called the $\alpha$-carbon. Their generic formula is $\mathrm{H}_{2} \mathrm{NCHRCOOH}$ where $\mathrm{R}$ is a generic organic group. These $\alpha$-amino acids are particularly important because they are the building blocks of proteins; 22 of them, biosynthesized from other molecules have been identified in living organisms. The simplest of all $\alpha$-amino acids is glycine, whose $\mathrm{R}$ group indeed consists of one $\mathrm{H}$ atom. Incidentally, because of its simple structure, glycine is the only $\alpha$-amino acid that is not chiral, in other words has no optical activity (the search for chirality in the ISM is addressed in Marloie et al. 2010).

Amino acids are not restricted to Earth. They have been found in great quantities (to form up to a few percent of the organic carbon; for a review see e.g. Sephton 2002) in several meteorites, specifically in those believed to be the most primitive ones, i.e. the carbonaceous chondrites. For example, more than 70 amino acids have been identified in the Murchison meteorite, including 8 out of the 22 standard amino acids present in living organisms (Cooper et al. 1992). Among them, glycine is one of the most abundant (see e.g. Botta et al. 2002). On the basis of this evidence, the consensus is that amino acids in meteorites form thanks to a multi-step process (Cronin et al. 1995) implying that amino acid precursors (aldehydes, ketones, ammonia, and $\mathrm{HCN}$ ) were present (or formed) in the protosolar nebula, and later incorporated into the asteroidal parent body. During aqueous alteration on the parent body, Strecker-cyanohydrin synthesis would have taken place to form the $\alpha$-amino acids (Peltzer et al. 1984; Cronin \& Chang 1993; Lerner et al. 1993). It has been argued that some of the aminoacids that have been found in the meteorites may have been synthesized in the ISM (Pizzarello et al. 2006) because of the high level of deuteration (Pizzarello \& Huang 2004, 2005), which is similar to that found in the ISM (Ceccarelli et al. 2007). However, the observed high level of deuteration only proves that the (smaller) ISM molecules, which the aminoacids are formed from during the aqueous alteration, are highly deuterated and not necessarily that the aminoacids were formed in the ISM (Ceccarelli \& Cernicharo 2011).

While all the above amino acid precursors have been detected in the ISM, the search for the amino acids themselves has so far been unsuccessful. The detection of glycine $\left(\mathrm{NH}_{2} \mathrm{CH}_{2} \mathrm{COOH}\right)$, the smallest of the $\alpha$-amino acids, has been the object of the largest number of investigations ever made for any prebiotic molecule. Two conformers of glycine, separated 
by $\sim 2 \mathrm{kcal} / \mathrm{mol},{ }^{1}$ have been observed in the laboratory (Lovas et al. 1995). After several fruitless searches, a comprehensive study of Orion and Sgr B2 carried out by Combes et al. (1996) gave an upper limit of the column densities for both conformers and showed that no greater improvement could be expected with single dish telescopes because the glycine signature was below the line confusion limit. Some years later, glycine was searched for in the direction of the low-mass protostar IRAS16293-2422 (Ceccarelli et al. 2000), where the problem of the line confusion is very much lower than for Orion and SgrB2 and, therefore, the hope of finding the glycine signature should be higher. However, this search also failed, in a similar way to successive searches toward Sgr B2(N-LMH) and OMC1 (Hollis et al. 2003a,b). In the same year, hopes were raised by the claim of glycine detection in three high-mass star-formation regions, Sgr B2(N-LMH), Orion KL, and W51 (Kuan et al. 2003). However, it was first disputed (Snyder et al. 2005) and finally rejected four years later (Jones et al. 2007; Cunningham et al. 2007). After a long hunt, glycine in the ISM remains elusive as ever.

In this report, we address the issue of why the hunt for glycine in the ISM is so difficult and suggest within the glycine family some other options that could be more easily detected. To this end, we use the results of Lattelais et al. (2009). Briefly, based on the observations towards astronomical sources available in the literature and new computations of the stability of the isomers of same generic chemical formula, it has been found that the most abundant isomers of the same chemical formula are the most stable, with only two exceptions over a sample of 32 isomeric forms. This finding has been summarized as the minimum energy principle (MEP). This can be seen as a simplification of the concept of the minimization of the Gibbs energy under thermodynamic equilibrium circumstances. We have indeed verified that, at the temperature of the ISM, including entropic terms to determine exact Gibbs free energies has a marginal impact on the values of the energy differences and, therefore, can be neglected accordingly (Lattelais et al. 2010). The MEP result is largely unexpected in the ISM, where chemistry is usually assumed to be controlled by kinetics. In principle, the abundance ratios of different isomers are expected to depend on the specific formation and destruction routes. Against these expectations, the analysis of the measured abundances of isomers of the same generic formula suggests that the stability energy is more important than other mechanisms (see the discussion in Lattelais et al. 2009). Nonetheless, it is important to emphasise that the relative abundance of the isomers of the same generic formula does not strictly follow a Boltzmann law. Therefore, the MEP result does not imply that interstellar chemistry is not kinetically driven. It does rather imply that the isomerization processes after the species formation are, for reasons not yet understood, more important than expected.

There are exceptions to the MEP, notably the methyl formate/acetic acid and dimethyl ether/ethanol systems, in which the higher energy isomer is by far the most abundant. Several reasons can be advanced to rationalize these exceptions involving different formation efficiencies in the mantle of the grains, in the gas phase or at the solid/gas interface (Lattelais et al. 2009). In this context, a recent study of the last hypothesis, coupling thermal programmed desorption (TPD) experiments with quantum numerical simulations, has shown that desorption of the less stable isomers from the ice is largely favoured, which is not excluding the possible role of the other mechanisms (Lattelais et al., in prep.).

\footnotetext{
1 For convenience: $1 \mathrm{kcal} / \mathrm{mol}=350 \mathrm{~cm}^{-1}=500 \mathrm{~K}$.
}

In the same way, it has been shown that formamide (Rubin et al. 1971), acetamide (Hollis et al. 2006), and aminoacetonitrile (Belloche et al. 2008) were the most stable species of their generic families (Lattelais et al. 2010). The relatively high abundance of the isocyanide derivatives with respect to their more stable cyano counterparts (in view of the large bonding energy differences) has been ascribed to non-thermal processes in different interstellar environments (Remijan et al. 2005).

In this work, we have computed the stability of all the conformers of every isomer of glycine to predict the form that is expected to be the most abundant and, therefore, the most likely to be detected in the ISM. In addition to neutral glycine, we also consider the protonated, ionic, anionic, and zwitterionic forms of glycine. According to the type of environment, glycine may indeed appear in different forms. For example, it seems obvious to consider that $\mathrm{NH}_{2} \mathrm{CH}_{2} \mathrm{COOH}$ should be the dominant form in the dense clouds, but under the effect of a radiation field, $\left[\mathrm{NH}_{2} \mathrm{CH}_{2} \mathrm{COOH}\right]^{+}$could be abundant. In the same way, one could consider $\mathrm{NH}_{3}^{+} \mathrm{CH}_{2} \mathrm{COOH}$ where $\mathrm{H}_{3}^{+}$is present and acts as protonating agent, in addition to $\mathrm{NH}_{2} \mathrm{CH}_{2} \mathrm{COO}^{-}$as the most probable form of negative ion. Where grains are covered with an icy mantle, it is the zwitterionic form $\mathrm{NH}_{3}^{+} \mathrm{CH}_{2} \mathrm{COO}^{-}$that should be dominant. All these possibilities are discussed here in the light of the MEP.

The methodology is summarized in Sect. 2 and the results for neutral glycine and its isomers are reported in Sect. 3, whereas those of the other derivatives are given in Sect. 4. The discussion of the consequences of our computations is presented in Sect. 5.

\section{Computational background}

Since the astrophysical conclusions that can be drawn from the present calculations rely essentially on the relative energies of the various species considered, particular attention was given to the methods used for their calculation. In this paper, we also reconsider our preliminary work (Lattelais et al. 2007) that was limited to density functional theory (DFT) calculations only, for the lowest energy conformations of the glycine isomers.

The strategy employed for the neutral species proceeds in two steps. A first approach used DFT within the hybrid B3LYP formalism (Becke 1993; Lee et al. 1988), coupled with two levels of basis sets: standard split-valence polarized $6-311 \mathrm{G}(\mathrm{d}, \mathrm{p})$ and correlation consistent cc-pVQZ. All conformers were studied for each molecule and each fully optimized structure was verified to be a stationary point by a vibrational analysis. In a second step, a final estimate of the energy differences between the isomers/conformers was calculated at the highly correlated $\operatorname{CCSD}(\mathrm{T}) / \mathrm{cc}-\mathrm{pVQZ}$ level of theory using the geometry and zero point vibrationnal energy (ZPE) obtained at the B3LYP level with the same basis set. The strategy employed for the ionic and protonated species was limited to the B3LYP/6-311(d,p) level since this level of theory was shown to be appropriate for comparing the energies of organic compounds in a previous report (Lattelais et al. 2010). In this same report, we gave a thorough discussion of the computing scheme (choice of basis sets, choice of functional, comparison between DFT and CCSD(T) values,...) that needs not be duplicated here. For a comprehensive presentation of DFT methods and their application to reproduce the structural properties, energies and spectroscopic parameters (vibrations, dipole moments,...) of organic molecules, the reader should refer to Lee \& Scuseria (1995) and Koch \& Holthausen (2001).

Special attention has been given to the treatment of the species with a negative charge namely, negative ions and 
Table 1. Computed relative stabilities $(\mathrm{kcal} / \mathrm{mol})$ and dipole moments (Debye) of glycine isomers including ZPE corrections.

\begin{tabular}{lcccccc}
\hline \hline Molecule & $\mu^{a}$ & $\mu_{\mathrm{A}}{ }^{a}$ & $\mu_{\mathrm{B}}{ }^{a}$ & $\mu_{\mathrm{C}}{ }^{a}$ & $\Delta E^{a}$ & $\Delta E^{b}$ \\
\hline $\mathrm{CH}_{3} \mathrm{NHCOOH}$ & 2.4 & -1.0 & -2.2 & 0.0 & 0.0 & 0.0 \\
$\mathrm{CH}_{3} \mathrm{OC}\left(\mathrm{NH}_{2}\right) \mathrm{O}$ & 2.4 & -0.4 & -2.3 & 0.5 & 4.7 & 4.9 \\
$\mathrm{NH}_{2} \mathrm{CH}_{2} \mathrm{COOH}$ & 1.2 & 1.1 & -0.5 & 0.0 & 10.6 & 8.8 \\
$\mathrm{HOCH}_{2} \mathrm{C}\left(\mathrm{NH}_{2}\right) \mathrm{O}$ & 4.4 & 3.4 & 2.8 & 0.0 & 12.0 & 10.3 \\
$\mathrm{HOCH}_{2} \mathrm{NHCHO}$ & 3.1 & -0.1 & 3.1 & -0.3 & 18.0 & 16.7 \\
$\mathrm{NH}_{2} \mathrm{CH}_{2} \mathrm{OCHO}$ & 1.7 & 0.7 & -1.2 & -1.0 & 18.1 & 17.7 \\
$\mathrm{NH}_{2} \mathrm{CH}(\mathrm{OH}) \mathrm{CHO}$ & 1.6 & 1.2 & -1.0 & 0.3 & 30.8 & 27.6 \\
$\mathrm{NH}_{2} \mathrm{CH}=\mathrm{C}(\mathrm{OH})_{2}$ & 1.3 & -1.2 & 0.4 & 0.0 & 33.1 & 30.7 \\
\hline
\end{tabular}

Notes. ${ }^{(a)}$ B3LYP/6-311G(d,p); ${ }^{(b)} \operatorname{CCSD}(\mathrm{T}) / \mathrm{cc}-\mathrm{pVQZ}$.

zwitterionic forms, and diffuse functions have been added to the basis set in this case. The polarizable continuum model (PCM) (Tomasi \& Persico 1994) has been employed to simulate the environment of zwitterionic compounds embedded in the ice mantles. Briefly, the zwitterion is included in a cavity the size of the union of spheres of appropriate radii centred on every atom of the molecule (including hydrogen atoms). The cavity is created inside a uniform medium characterized by a dielectric constant that is a function of temperature. A value of 600 , extrapolated from the results obtained in a comprehensive study of the dielectric constant of water as a function of temperature (Johari \& Jones 1976; Johari \& Whalley 1981) was employed in the present modeling of the interstellar ices at $(10-50 \mathrm{~K})$, instead of the usual value of 80 for liquid water. We also computed the dipole moments along the main rotation axis for each isomer of neutral glycine, even though we are aware that they are of limited help to radio-astronomers, as the spectrum of molecules with several functional groups is complex and by no means predictible based on dipole moments only.

All calculations were performed using the GAUSSIAN03 package (Frisch et al. 2004).

\section{Neutral glycine and isomers}

A systematic investigation of the molecules of prebiotic interest with chemical formula $\mathrm{C}_{2} \mathrm{H}_{5} \mathrm{O}_{2} \mathrm{~N}$ led to the relative energies reported in Table 1 for the most stable conformer of each isomer. The cyclic isomers and those containing an NO bond were not considered for energetic reasons (Chiaramello et al. 2005; Lattelais et al. 2010).

The high level Coupled Cluster CCSD(T)/cc-pVQZ calculations agree with the B3LYP energy ordering (Lattelais et al. 2007), the energy differences being slightly overestimated in DFT. Our present study clearly finds that the most stable isomer is not glycine, but $\mathrm{N}$-methylcarbamic acid, $\mathrm{CH}_{3} \mathrm{NHCOOH}$. To the best of our knowledge, this isomer with a dipole moment of 2.4 Debye has never been searched for in the ISM and the frequencies of its rotational transitions are not known. Second in terms of energy is methyl carbamate $\mathrm{CH}_{3} \mathrm{OC}\left(\mathrm{NH}_{2}\right) \mathrm{O}$, which has also a dipole moment of $\sim 2.4$ Debye. The most stable conformer of glycine, known as conformer I, ranks third on the energy scale, at $\sim 10 \mathrm{kcal} / \mathrm{mol}$ above the most stable structure. In the context of possible detection, we note that molecules containing carboxylic $(-\mathrm{COOH})$ or amide $\left(-\mathrm{CONH}_{2}\right)$ groups are more stable than those with the aldehydic (-CHO) group.

However, we recall that the detection of molecules in radio relies for a large part on the size of their dipole moment. It is
Table 2. Computed relative stabilities $(\mathrm{kcal} / \mathrm{mol})$ and dipole moments (Debye) of Glycine conformers at the B3LYP/6-311G(d,p) level including ZPE corrections.

\begin{tabular}{|c|c|c|c|c|c|}
\hline Conformer & $\mu$ & $\mu_{\mathrm{A}}$ & $\mu_{\mathrm{B}}$ & $\mu_{\mathrm{C}}$ & $\Delta E$ \\
\hline & 1.2 & 1.1 & -0.5 & 0.0 & 0.0 \\
\hline & 5.6 & -5.5 & -0.8 & 0.0 & 0.8 \\
\hline & 1.9 & 0.3 & 1.8 & 0.0 & 1.6 \\
\hline & 2.0 & -0.2 & -1.5 & 1.4 & 1.8 \\
\hline & 2.4 & 1.5 & 1.1 & 1.5 & 3.3 \\
\hline & 2.9 & -0.4 & -2.9 & 0.0 & 5.5 \\
\hline & 4.3 & -4.1 & 1.1 & 0.0 & 6.2 \\
\hline & 4.0 & 1.1 & -3.7 & 1.3 & 7.3 \\
\hline
\end{tabular}

then worth asking the question of whether conformers of higher energy could be more easily detected because of larger dipole moments. The case of glycine is particularly illustrative (see Table 2). The first spectrum observed in the laboratory (Brown et al. 1978; Suenram \& Lovas 1978) was that of a higher conformer (later referred to as conformer II) because its dipole moment of $\sim 5.6$ Debye is much higher than that of the most stable isomer ( 1.2 Debye). Conformer I was later characterized (Suenram \& Lovas 1980) and the energy difference estimated to be $\sim 2 \mathrm{kcal} / \mathrm{mol}$ together with dipole moments of 0.9 and 5.4 debye for comformers I and II, respectively (Lovas et al. 1995).

Seven conformers of glycine can be found within $\sim 7 \mathrm{kcal} / \mathrm{mol}$ of conformer I; the relative energies and structures of five, with whom the present calculations agree, were reported by Holtom et al. (2005). The relative energies and dipole components of conformers I to VIII are presented in Table 2 (structural details being given in Appendix A). It is unfortunate that the most stable conformer is the one with the smallest dipole moment. None of the high energy conformers has a dipole moment larger than that of conformer II and therefore they are of no help in the detection of glycine.

A similar study has been performed on the low-lying isomers of glycine, $\mathrm{CH}_{3} \mathrm{NHCOOH}$ and $\mathrm{CH}_{3} \mathrm{OC}\left(\mathrm{NH}_{2}\right) \mathrm{O}$, and the results are presented in Tables 3 and 4 (structural details are given in Appendix B). Four conformers of N-methylcarbamic acid can be found within a $\sim 9 \mathrm{kcal} / \mathrm{mol}$ energy range. The lowest two conformers, separated by $1.5 \mathrm{kcal} / \mathrm{mol}$ have similar dipole 
Table 3. Computed relative stabilities $(\mathrm{kcal} / \mathrm{mol})$ and dipole moments (Debye) of N-methylcarbamic acid conformers at the B3LYP/6$311 \mathrm{G}(\mathrm{d}, \mathrm{p})$ level including ZPE corrections.

\begin{tabular}{lllllll} 
& $\mu$ & $\mu_{\mathrm{A}}$ & $\mu_{\mathrm{B}}$ & $\mu_{\mathrm{C}}$ & $\Delta E$ \\
\hline & & & & & & \\
\hline
\end{tabular}

Table 4. Computed relative stabilities $(\mathrm{kcal} / \mathrm{mol})$ and dipole moments (Debye) of Methylcarbamate conformers at the B3LYP/6-311G(d,p) level including ZPE corrections.

\begin{tabular}{lllllll}
\hline \hline Conformer & $\mu$ & $\mu_{\mathrm{A}}$ & $\mu_{\mathrm{B}}$ & $\mu_{\mathrm{C}}$ & $\Delta E$ \\
\hline & & & & & & \\
\hline
\end{tabular}

moments, i.e. $\mu \sim 2.5$ Debye. The other two conformers have more significant dipole moments of $\sim 5$ Debye; however, they are of higher energy $(\sim 8-9 \mathrm{kcal} / \mathrm{mol})$ and less likely to be of large enough abundance for detection. The second low-lying isomer, methyl carbamate, has also a dipole moment of $\mu \sim 2.5$ Debye. For that reason, it has been tentatively searched for in the hot molecular cloud W51e 2 and in the intermediate mass protostar IRAS21391+58502 (Demyk et al. 2004). Its millimeter and submillimeter wave spectrum has been the object of an in depth study that should now allow this isomer of glycine to be searched for over a wider frequency range (Groner et al. 2007). The second conformer of methyl carbamate with significant $\mu \sim 5$ Debye is $8 \mathrm{kcal} / \mathrm{mol}$ higher on the energy scale. Under these circumstances, it seems clear that the lowest energy conformers of the lowest energy isomers are the most plausible targets for radio detection.

\section{Other forms of glycine and isomers}

\subsection{Protonated glycine}

In an environment where $\mathrm{H}_{3}^{+}$is abundant, proton transfer reactions are common processes. Most of the positive ions in the ISM are protonated species, $\mathrm{HCO}^{+}, \mathrm{HOC}^{+}, \mathrm{HCNH}^{+}$and some, such as $\mathrm{NNH}^{+}$or $\mathrm{H}_{3} \mathrm{O}^{+}$, are used to probe their precursor neutral molecules $\left(\mathrm{N}_{2}\right.$ and $\left.\mathrm{H}_{2} \mathrm{O}\right)$ which are otherwise difficult to observe. For this reason, we checked all the protonated isomers
Table 5. Computed relative stabilities $(\mathrm{kcal} / \mathrm{mol})$ and dipole moments (Debye) of protonated glycine isomers at the B3LYP/6-311G $(\mathrm{d}, \mathrm{p})$ level including ZPE corrections.

\begin{tabular}{lcc}
\hline \hline Molecule & $\mu$ & $\Delta E$ \\
\hline $\mathrm{NH}_{3}^{+} \mathrm{CH}_{2} \mathrm{COOH}$ & 5.6 & 0.0 \\
$\mathrm{CH}_{3} \mathrm{NHC}(\mathrm{OH})_{2}^{+}$ & 1.4 & 2.2 \\
$\mathrm{CH}_{3} \mathrm{OC}\left(\mathrm{NH}_{2}\right) \mathrm{OH}^{+}$ & 1.9 & 4.7 \\
$\mathrm{HOCH}_{2} \mathrm{C}\left(\mathrm{NH}_{2}\right) \mathrm{OH}^{+}$ & 3.2 & 6.0 \\
$\mathrm{NH}_{3}^{+} \mathrm{CH}_{2} \mathrm{OCHO}$ & 5.7 & 15.4 \\
$\mathrm{HOCH}_{2} \mathrm{NHCHOH}$ & 3.2 & 16.0 \\
$\mathrm{NH}_{3}^{+} \mathrm{CH}=\mathrm{C}(\mathrm{OH})_{2}$ & 4.6 & 19.9 \\
$\mathrm{NH}_{3}^{+} \mathrm{CH}(\mathrm{OH}) \mathrm{CHO}$ & 3.6 & 27.9 \\
\hline
\end{tabular}

Table 6. Computed relative stabilities ( $\mathrm{kcal} / \mathrm{mol}$ ) and dipole moments (Debye) of glycine cations isomers at the B3LYP/6-311G(d,p) level including ZPE corrections.

\begin{tabular}{lcc}
\hline \hline Molecule & $\mu$ & $\Delta E$ \\
\hline $\mathrm{NH}_{2} \mathrm{CH}=\mathrm{C}(\mathrm{OH})_{2}^{+}$ & 2.2 & 0.0 \\
$\mathrm{HOCH}=\mathrm{C}\left(\mathrm{NH}_{2}\right) \mathrm{OH}^{+}$ & 3.0 & 3.6 \\
$\mathrm{HOCHCO}\left(\mathrm{NH}_{3}\right)^{+}$ & 4.4 & 15.8 \\
$\mathrm{NH}_{3}^{+} \mathrm{CHCOOH}$ & 6.2 & 17.7 \\
$\mathrm{NH}_{2} \mathrm{CH}_{2} \mathrm{COOH}^{+}$ & 4.3 & 21.7 \\
$\mathrm{CH}_{3} \mathrm{NHCOOH}^{+}$ & 3.9 & 25.2 \\
$\mathrm{HOCH}_{2} \mathrm{C}\left(\mathrm{NH}_{2}\right) \mathrm{O}^{+}$ & 2.2 & 30.6 \\
$\mathrm{CH}_{3} \mathrm{~N}=\mathrm{C}(\mathrm{OH})_{2}^{+}$ & 1.0 & 33.9 \\
\hline
\end{tabular}

that can be formed from the $[2 \mathrm{C}, 5 \mathrm{H}, 2 \mathrm{O}, \mathrm{N}] \mathrm{H}^{+}$distribution. Different possible sites of protonation were considered for each glycine isomer.

The result is somewhat surprising: the isomer of protonated glycine, $\left(\mathrm{NH}_{3}^{+} \mathrm{CH}_{2} \mathrm{COOH}\right)$, is now the most stable, unlike its neutral parent (structural details are given in Appendix C), as shown in Table 5. Protonation on the oxygen atoms of glycine leads to either the transfer of the proton to the nitrogen atom or dissociation. The three lowest energy isomers are the same as the neutral compounds, but the ordering is different and they are closer together on the energy scale, i.e. within $\sim 5 \mathrm{kcal} / \mathrm{mol}$ instead of about 10 previously. Protonation increased the dipole moment of glycine by a factor of about 5 making it an attractive candidate.

\subsection{Positive and negative ion isomers of glycine}

An environment with a high radiative field would result in the ionization of glycine and maybe its destruction. The relative energies of the $\left[\mathrm{C}_{2} \mathrm{H}_{5} \mathrm{O}_{2} \mathrm{~N}\right]^{+}$isomers are given in Table 6 . The most stable ion is the enolic cation of glycine $\left[\mathrm{NH}_{2} \mathrm{CH}=\mathrm{C}(\mathrm{OH})_{2}\right]^{+}$ (structural details are given in Appendix C). Ionized glycine is found in fifth position, $\sim 1 \mathrm{eV}$ above its enolic form. It is worth noting that the most stable ions are the products of proton transfers and not the ion obtained by simple ionization. More importantly, this enolic ion is prochiral, which means that it will give a chiral molecule by addition of a substituent to the $\alpha$-carbon (other than $\mathrm{H}$ ).

A number of negative ions have been observed in the ISM, which were produced after electron capture by conjugated 
Table 7. Computed relative stabilities $(\mathrm{kcal} / \mathrm{mol})$ and dipole moments (Debye) of glycine anions isomers at the B3LYP/6-311+G(d,p) level including ZPE corrections.

\begin{tabular}{lcc}
\hline \hline Molecule & $\mu$ & $\Delta E$ \\
\hline $\mathrm{CH}_{3} \mathrm{NHCOO}^{-}$ & 5.7 & 0.0 \\
$\mathrm{NH}_{2} \mathrm{CH}_{2} \mathrm{COO}^{-}$ & 3.9 & 3.3 \\
$\left.\mathrm{HOCH}_{2} \mathrm{C}(\mathrm{NH})\right)^{-}$ & 1.5 & 13.8 \\
$\mathrm{CH}_{3} \mathrm{OC}(\mathrm{NH}) \mathrm{O}^{-}$ & 3.7 & 19.0 \\
$\mathrm{HOCH}_{2} \mathrm{NCHO}^{-}$ & 2.1 & 21.9 \\
$\mathrm{NH}_{2} \mathrm{CH}\left(\mathrm{O}^{-}\right) \mathrm{CHO}$ & 2.5 & 38.2 \\
$\mathrm{NH}_{2}(\mathrm{CH})^{-} \mathrm{OCHO}$ & 2.2 & 60.6 \\
\hline
\end{tabular}

systems. In the case of glycine, the most probable way of forming a negative ion is not the electron capture but the abstraction of a proton (Table 7). The most stable isomer is $\mathrm{CH}_{3} \mathrm{NHCOO}^{-}$, which is related not to glycine but to N-methyl carbamic acid the most stable in neutral form (structural details are given in Appendix C). The glycine carboxylate ion is $\sim 3.3 \mathrm{kcal} / \mathrm{mol}$ higher in energy.

All of the above molecules are pertinent to gas phase observations. However, it is well known that dust and grains play an important role in interstellar chemistry. When formed on the icy surfaces of the grains or attached to them by hydrogen bonds, glycine does not remain in any of the forms discussed previously. It has been shown in the laboratory that glycine is present under a zwitterionic form $\mathrm{NH}_{3}^{+} \mathrm{CH}_{2} \mathrm{COO}^{-}$in the ice (e.g. Holtom et al. 2005), this intramolecular ion pair being unstable in the gas phase. Only N-methyl carbamic acid can produce another viable ion pair, $\mathrm{CH}_{3} \mathrm{NH}_{2}^{+} \mathrm{COO}^{-}$but it is less stable by $\sim 3.3 \mathrm{kcal} / \mathrm{mol}$ than that of glycine which should be the major product in these conditions (structural details are given in Appendix D).

\section{Concluding remarks}

The goal of the presented work has been to determine the best isomers of glycine to search for in the ISM, namely those that would have the highest abundance based on the MEP described in the Introduction. First, and probably most important, our high level CCSD(T) calculations confirm the preliminary results (Lattelais et al. 2007) showing that glycine is not the most stable form of the generic formula $\mathrm{C}_{2} \mathrm{H}_{5} \mathrm{O}_{2} \mathrm{~N}$. It is $\mathrm{N}$-methyl carbamic acid that is the most stable isomer and, in addition, has a dipole moment twice as large, so that its detection should be easier (or more probable) for both reasons. Various laboratory experiments that have studied the formation of glycine in simulated interstellar ices support our findings. For example, thermal reactions or reactions triggered by energetic particle bombardment end up forming $\mathrm{N}$-methyl carbamic acid rather than glycine (e.g. Holtom et al. 2005; Bossa et al. 2008). Only successive UV irradiation would then form glycine (Bossa et al. 2009). However, one should notice that both N-methyl carbamic acid and glycine have a carboxylic group with a free acidic hydrogen available for hydrogen bonding, which may lead to a possible reduction in their abundance with respect to what is predicted on the sole basis of MEP. Methyl carbamate, $\mathrm{CH}_{3} \mathrm{OC}\left(\mathrm{NH}_{2}\right) \mathrm{O}$, has no such drawback; it is lower in energy than glycine and also has a larger dipole moment that makes it a worthy target as suggested also by Garrod et al. (2008) on the basis of its formation chemistry. Although neither N-methyl carbamic acid nor methyl carbamate have the precious structure of glycine with the amine and the carboxyl groups, which, via the peptide bonds, form the long chains needed by the genetic code to pass information, discovering them in the ISM would prove that prebiotic chemistry can take place in interstellar space even in the absence of liquid water.

Another possible way of trying to detect glycine is to search for its ionic, protonated and zwitterionic forms. We have shown that, under a cationic or anionic form, glycine is also not the most stable compound. Ionization gives a highly energetic compound that would hardly survive as shown by recent experiments at the SOLEIL synchrotron facility (Lattelais et al. 2011). The loss of a proton leads to the basic form of glycine, which is close in energy to that of the N-methyl carbamic acid although the latter negative ion remains a better candidate. Glycine is the most stable compound only under a protonated form in the gas phase with a dipole moment of $~ 5.6$ Debye. However, its abundance depends on the electron density of the medium since recombination with an electron would destroy the molecular backbone but not remove the proton. Finally, we have shown that zwitterionic glycine is the most stable isomer on the ices, which leaves the door open for a possible identification in solids, for example in cometary ices ${ }^{2}$ but not in the gas phase where this ion-pair cannot survive.

In summary, when considering the stability energy of all the possible isomers (and conformers) of the generic chemical formula $\mathrm{C}_{2} \mathrm{H}_{5} \mathrm{O}_{2} \mathrm{~N}$ and its ionic, protonated, and zwitterionic variants, it turns out that glycine is not the most stable isomer. Based on the minimum energy principle (see Sect. 1), this implies that glycine is probably not the most abundant isomer either and, therefore, more difficult to detect in the ISM than other more stable isomers. We note that the protonated glycine is predicted to be the most stable isomer of the family, although its abundance is expected to be very low. On the other hand, it will certainly be important to test whether the two more stable isomers of glycine, N-methylcarbamic, acid and methyl carbamate, are present in the ISM. If so, this will be an indication that a first but important step towards prebiotic chemistry may take place in the ISM.

Acknowledgements. This work was supported by CNRS national programs PCMI (Physics and Chemistry of the Interstellar Medium), OPV (Origins of Planets and Life) and the Agence Nationale pour la Recherche (ANR), France (contract ANR-08-BLAN-0225, project FORCOMS). Part of the calculations were performed using HPC resources from GENCI-CINES (Grant 2009085128).

\section{Appendix A:}

Figure A.1 shows selected structural parameters of the glycine conformers (Table 2), as obtained after full geometry optimization at the B3LYP/6-311(d,p) level of theory.

\section{Appendix B:}

Figure B.1 shows selected structural parameters of the N-methylcarbamic acid (Table 3) and Methylcarbamate (Table 4) conformers, as obtained after full geometry optimization at the B3LYP/6-311(d,p) level of theory.

\section{Appendix C:}

Figure C.1 shows selected structural parameters of the lowest energy conformers of the protonated glycine (Table 5), the enol

\footnotetext{
${ }^{2}$ See e.g. J. Elsila et al. http://www.spaceref.com/news/ viewpr.html
} 


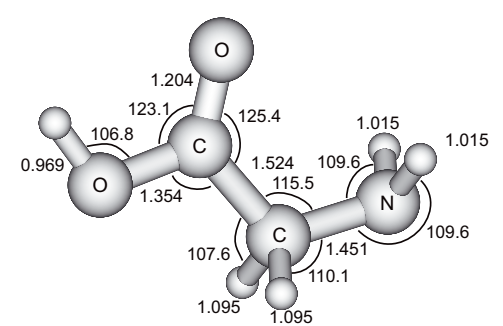

(a)

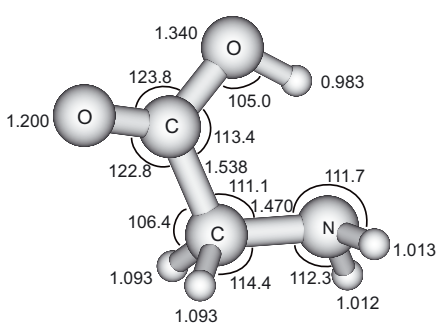

(b)

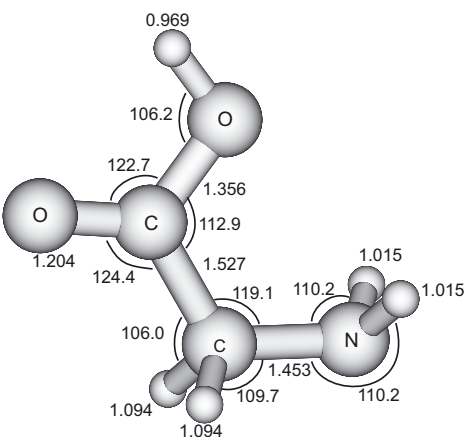

(c)

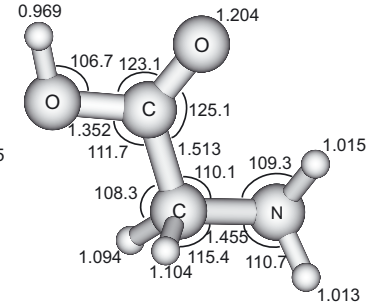

(d)

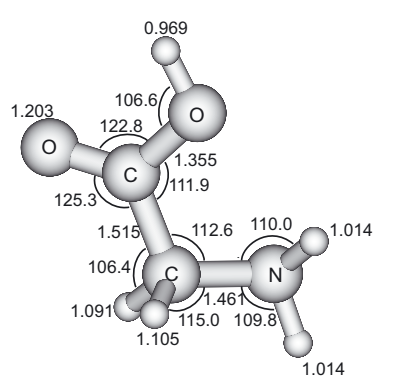

(e)

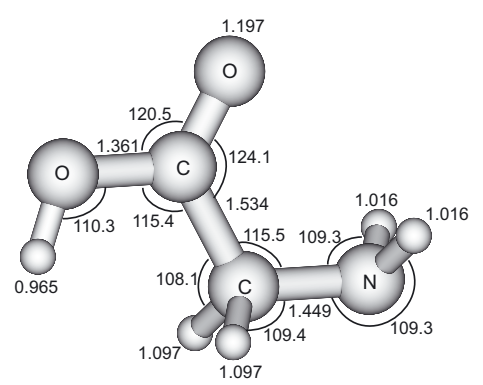

(f)

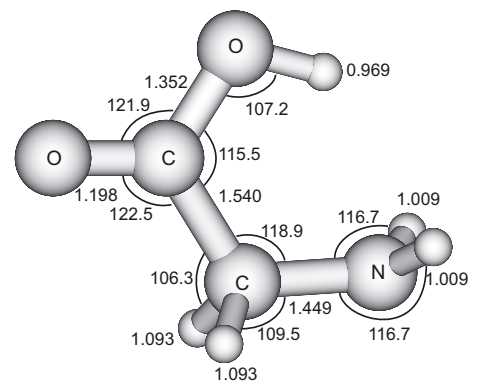

(g)

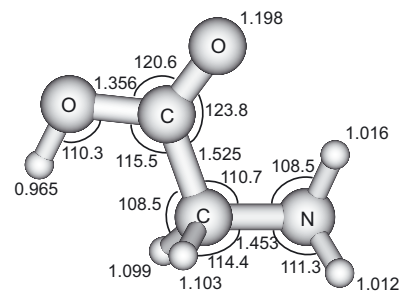

(h)

Fig. A.1. Structures ( $\AA$; deg) of glycine conformers.

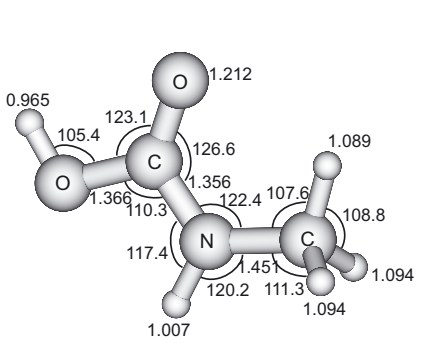

(a)

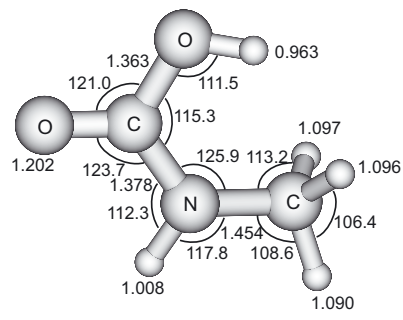

(d)

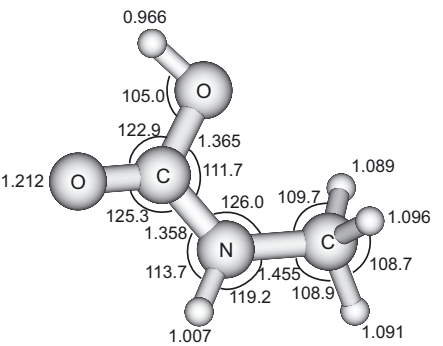

(b)

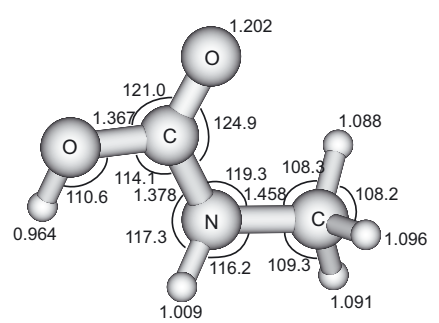

(c)

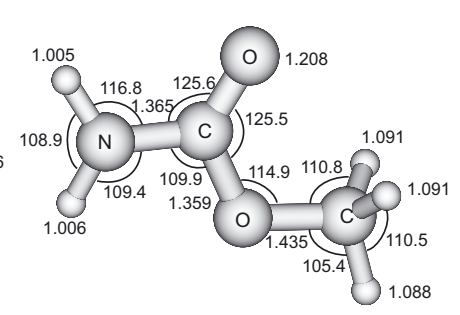

(e)

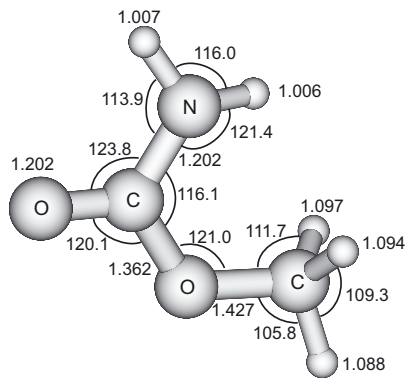

(f)

Fig. B.1. Structures ( $\AA$; deg) of N-methylcarbamic acid conformers a)-d) and Methylcarbamate conformers e)-f).

of ionized glycine (Table 6) and the anion of N-methylcarbamic acid (Table 7), as obtained after full geometry optimization at the B3LYP/6-311(d,p) level of theory for the positive ions and B3LYP/6-311+(d,p), i.e., with diffuse functions for the negative ion.

\section{Appendix D:}

Figure D.1 shows selected structural parameters of the lowest energy conformers of the zwitterionic forms of N-methylcarbamic acid (a) and glycine (b), as obtained after full geometry 


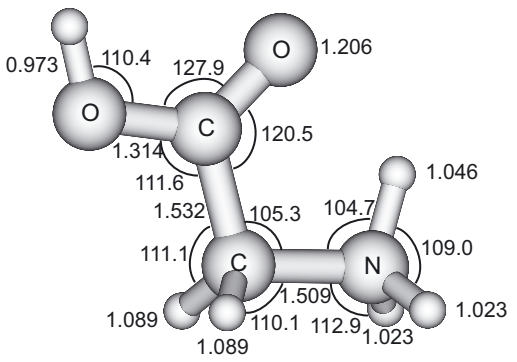

(a)

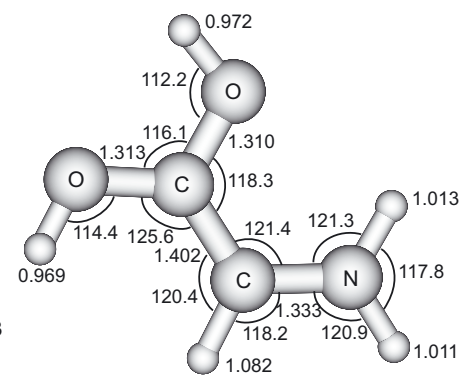

(b)

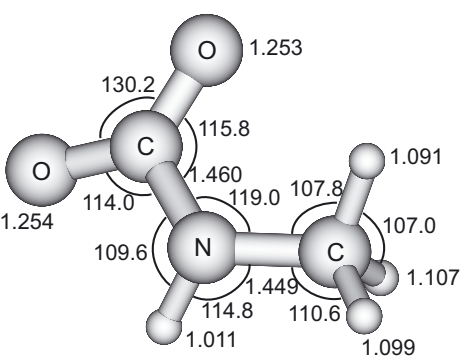

(c)

Fig. C.1. Structures ( $\AA$; deg) of protonated glycine a), ionized glycine b) and N-methylcarbamic anion c).

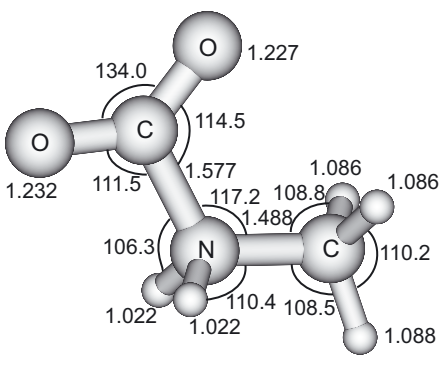

(a)

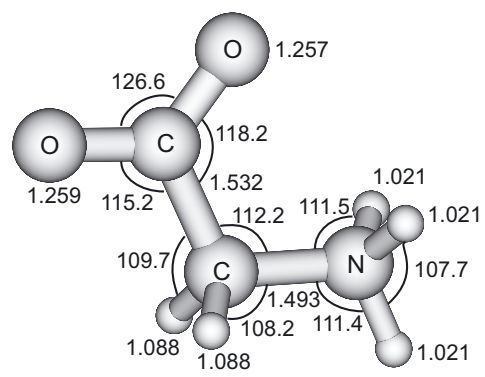

(b)

Fig. D.1. Structures ( $\AA$; deg) of N-methylcarbamic acid a) and glycine b) zwitterions.

optimization at the B3LYP/6-311+(d,p) level of theory with diffuse functions.

\section{References}

Becke, A. D. 2000, J. Chem. Phys., 98, 5648

Belloche, A., Menten, K. M., Comito, C., et al. 2008, A\&A, 482, 179

Bossa, J.-B., Borget, F., Duvernay, F., Theulé, P., \& Chiavassa, T. 2008, J. Phys. Chem. A, 112, 5113

Bossa, J.-B., Duvernay, F., Theulé, P., et al. 2009, A\&A, 506, 601

Botta, O., Glavin, D. P., Kminek, G., \& Bada, J. L. 2002, Origins of Life and Evolution of the Biosphere, 32, 143

Brown, R. D., Godfrey, P. D., Storey, J. W. V., \& Bassez, M.-P. 1978, J. Chem. Soc. Chem. Comm., 547

Ceccarelli, C., \& Cernicharo, J. 2011, Origins and Evolution of Life - An Astrobiological Perspective, Gargaud, ed. P. Lopez-Garcia, \& H. Martin (Cambridge, USA: Cambridge University Press), 85

Ceccarelli, C., Loinard, L., Castets, A., Faure, A., \& Lefloch, B. 2000. A\&A, 362,1122

Ceccarelli, C., Caselli, P., Herbst, E., Tielens, A. G. G. M., \& Caux, E. 2007, Protostars and Planets V, ed. B. Reipurth, D. Jewitt, \& K. Keil (University of Arizona Press, Tucson, USA), 47

Chiaramello, J. M., Talbi, D., Berthier, G., \& Ellinger, Y. 2005, Int. J. Astrobiol., 4, 125

Cooper, G. W., Onwo, W. M., \& Cronin, J. R. 1992, Geochim. Cosmochim. Acta, 54, 4109

Combes, F., Nguyen-Q-Rieu, \& Wlodarczak, G. 1996, A\&A, 308, 618

Cronin, J. R., \& Chang, S. 1993, The Chemistry of Life Origin, ed. J. M. Greenberg, et al. (The Nederlands, Dordrecht: Kluwer), 209

Cronin, J. R., Cooper, G. W., \& Pizzarello, S. 1995, Adv. Space Res., 15, 91

Cunningham, M. R., Jones, P. A., Godfrey, P. D., et al. 2007, MNRAS, 376, 1201

Demyk, K., Wlodarczak, G., \& Dartois, E. 2004, in Semaine de l'Astrohysique Francaise, ed. F. Combes, D. Barret, T. Contini, F. Meynadier, \& L. Pagani, SF2A-2004 (Les Ulis: EDP-Sciences), 493

Frisch, M., Trucks, G., \& Schlegel, H. 2004, Gaussian 03, Rev.C.02, Gaussian Inc., Wallingford CT., 611, 605

Garrod, R. T., Widicus Weaver, S. L., \& Herbst, E. 2008, ApJ, 682, 283

Groner, P., Winnewisser, M., Medvedev, I. R., et al. 2007, ApJ, 169, 28

Hollis, J. M., Pedelty, J. A., Snyder, L. E., et al. 2003a, ApJ, 588, 353

Hollis, J. M., Pedelty, J. A., Snyder, L. E., et al. 2003b, ApJ, 596, L235
Hollis, J. M., Lovas, F. J., Remijan, A. J., et al. 2006, ApJ, 643, L25

Holtom, P. D., Bennett, C. J., Osamura, Y., Mason, N. J., \& Kaiser, R. I. 2005, ApJ, 626, 940

Johari, G. P., \& Jones, S. J. 1976, Proc. Roy. Soc. A, 349, 467

Johari, G. P., \& Whalley, E. J. 1981, J. Chem. Phys., 75, 1333

Jones, P. A., Cunningham, M. R., Godfrey, P. D., \& Cragg, D. M. 2007, MNRAS, 374,579

Koch, W., \& Holthausen, M. C. 2001, A Chemist Guide to Density Functional Theory (Weinheim, Germany: Wiley-VCH) 2nd edn.

Kuan, Y.-J., Charnley, S. B., Huang, H.-C., et al. 2003, ApJ, 593, 848

Lattelais, M., Ellinger, Y., \& Zanda, B. 2007, Int. J. Astrobiol., 6, 37

Lattelais, M., Pauzat, F., Ellinger, Y., \& Ceccarelli, C. 2009, ApJ, 696, L133

Lattelais, M., Pauzat, F., Ellinger, Y., \& Ceccarelli, C. 2010, A\&A, 519, A30

Lattelais, M., Risset, O., Pilmé, J., et al. 2011, Int. J. Quantum Chem., 111, 1163

Lee, T. J., \& Scuseria, G. E. 1995, in Quantum mechanical electronic structure calculations with chemical accuracy, ed. S. R. Langhoff (Dordrecht, The Netherlands: Kluwer Academic Publishers)

Lee, C., Yang, W., \& Parr, R. G. 1988, Phys. Rev. B, 37, 785

Lerner, N. R., Peterson, E., \& Chang, S. 1993, Geochim. Cosmochim. Acta, 57, 4713

Lovas, F. J., Kawashima, Y., Grabow, J.-U., et al. 1995, ApJ, 455, L201

Marloie, G., Lattelais, M., Pauzat, F., \& Ellinger, Y. 2010, Interdiscip. Sci. Comput. Life Sci., 2, 1

Peltzer, E. T., Bada, J. L., Schlesinger, G., et al. 1984, Adv. Space Res., 4, 69

Pizzarello, S., \& Huang, Y., 2004, in Proc. 35th Annual Lunar and Planetary Science, 1212

Pizzarello, S., \& Huang, Y., 2005, Geochim. Cosmochim. Acta, 49, 599

Pizzarello, S., Cooper, G. W., \& Flynn, G. J., 2006, Meteorites and the early solar system II, ed. D. S. Lauretta, \& H. Y. McSween Jr (Tucson, USA: University of Arizona Press), 625

Remijan, A. R., Hollis, J. M., Lovas, F. J., Plusquellic, D. F., \& Jewell, P. R. 2005, ApJ, 632, 333

Rubin, R. H., Swenson, Jr., G. W., Benson, R. C., Tigelaar, H. L., \& Flygare, W. H. 1971, ApJ, 169, L39

Sephton, M. A. 2002, Nat. Prod. Rep., 19, 292

Snyder, L. E., Lovas, F. J., Hollis, J. M., et al. 2005, ApJ, 619, 914

Suenram, R. D., \& Lovas, F. J. 1978, J. Mol. Spectr., 72, 372

Suenram, R. D., \& Lovas, F. J. 1980, J. Am. Chem. Soc., 102, 7180 ApJ, 455, L201

Tomasi, J., \& Persico, M. 1994, Chem. Rev., 94, 2027 